\title{
A GENERAL $q$-EXPANSION FORMULA BASED ON MATRIX INVERSIONS AND ITS APPLICATIONS
}

\author{
JIN WANG † \\ Department of Mathematics, Soochow University \\ Suzhou 215006, P. R. China \\ Email: jinwang2016@yahoo.com
}

\begin{abstract}
In this paper, by use of matrix inversions, we establish a general $q$-expansion formula of arbitrary formal power series $F(z)$ with respect to the base

$$
\left\{z^{n} \frac{(a z ; q)_{n}}{(b z ; q)_{n}} \mid n=0,1,2 \cdots\right\} .
$$

Some concrete expansion formulas and their applications to $q$-series identities are presented, including Carlitz's $q$-expansion formula, a new partial theta function identity and a coefficient identity of Ramanujan's ${ }_{1} \psi_{1}$ summation formula as special cases.
\end{abstract}

\section{INTRODUCTION}

Throughout the present paper, we adopt the standard notation and terminology for $q$-series from the book [7. As customary, the $q$-shifted factorials of complex variable $z$ with the base $q:|q|<1$ are given by

$$
(z ; q)_{\infty}:=\prod_{n=0}^{\infty}\left(1-z q^{n}\right) \quad \text { and } \quad(z ; q)_{n}:=\frac{(z ; q)_{\infty}}{\left(z q^{n} ; q\right)_{\infty}}
$$

for all integers $n$. For integer $m \geq 1$, we use the multi-parameter compact notation

$$
\left(a_{1}, a_{2}, \ldots, a_{m} ; q\right)_{n}:=\left(a_{1} ; q\right)_{\infty}\left(a_{2} ; q\right)_{n} \ldots\left(a_{m} ; q\right)_{n} .
$$

Also, the ${ }_{r+1} \phi_{r}$ series with the base $q$ and the argument $z$ is defined to be

$$
{ }_{r+1} \phi_{r}\left[\begin{array}{c}
a_{1}, a_{2}, \cdots, a_{r+1} \\
b_{1}, b_{2}, \cdots, b_{r}
\end{array} ;, z\right]:=\sum_{n=0}^{\infty} \frac{\left(a_{1}, a_{2}, \ldots, a_{r+1} ; q\right)_{n}}{\left(q, b_{1}, b_{2}, \ldots, b_{r} ; q\right)_{n}} z^{n} .
$$

For any $f(z)=\sum_{n>0} a_{n} z^{n} \in \mathbb{C}[[z]], \mathbb{C}[[z]]$ denotes the ring of formal power series in variable $z$, we shall employ the coefficient functional

$$
\left[z^{n}\right]\{f(z)\}:=a_{n} \text { and } a_{0}=f(0) .
$$

We also follow the summation convention that for any integers $m$ and $n$,

$$
\sum_{k=m}^{n} a_{k}=-\sum_{k=n+1}^{m-1} a_{k}
$$

2010 Mathematics Subject Classification. Primary 33D15 ; Secondary 05A30.

Key words and phrases. q-Series; Expansion formula; Coefficient; Transformation; Summation; Matrix inversion; Lagrange-Bürmann inversion; Formal power series. 
In their paper 4, G.H. Coogan and K. Ono presented the following identity which leads to the generating functions for values of certain expressions of Hurwitz zeta functions at non-positive integers.

Lemma 1.1 (cf. [4, Proposition 1.1]). For $|z|<1$, it holds

$$
\sum_{n=0}^{\infty} z^{n} \frac{(z ; q)_{n}}{(-z ; q)_{n+1}}=\sum_{n=0}^{\infty}(-1)^{n} z^{2 n} q^{n^{2}} .
$$

The appearance of (1.2) reminds us of the famous Rogers-Fine identity [11, Eq. (17.6.12)].

Lemma 1.2. For $|z|<1$, it holds

$$
(1-z) \sum_{n=0}^{\infty} z^{n} \frac{(a q ; q)_{n}}{(b q ; q)_{n}}=\sum_{n=0}^{\infty}\left(1-a z q^{2 n+1}\right)(b z)^{n} q^{n^{2}} \frac{(a q, a z q / b ; q)_{n}}{(b q, z q ; q)_{n}}
$$

In fact, Identity (1.2) can be easily deduced from (1.3) via setting $a q=z=-b$. Moreover, by setting $a=z=-b$ in (1.3), we obtain another similar identity.

Lemma 1.3. For $|z|<1$, it holds

$$
\sum_{n=0}^{\infty} z^{n} \frac{(z ; q)_{n+1}}{(-z q ; q)_{n}}=1+2 \sum_{n=1}^{\infty}(-1)^{n} z^{2 n} q^{n^{2}}
$$

It is these identities, once treated as formal power series in $z$, that make us be aware of investigating in a full generality the problem of representations of formal power series in terms of the sequences

$$
\left\{z^{n} \frac{(a z ; q)_{n}}{(b z ; q)_{n}} \mid n=0,1,2 \cdots\right\}
$$

which is just a base of the ring $\mathbb{C}[[z]]$. This fact asserts that for any $F(z) \in \mathbb{C}[[z]]$, there exists the series expansion

$$
F(z)=\sum_{n=0}^{\infty} c_{n} z^{n} \frac{(a z ; q)_{n}}{(b z ; q)_{n}}
$$

where the coefficients $c_{n}$ must be independent of $z$ but may depend on the parameters $a$ and $b$. In this respect, particularly noteworthy is that in [10, X.R. Ma established a (formal) generalized Lagrange-Bürmann inversion formula. We record it for direct reference.

Lemma 1.4 (cf. [10, Theorem 2.1]). Let $\left\{\phi_{n}(z)\right\}_{n=0}^{\infty}$ be arbitrary sequence of formal power series with $\phi_{n}(0)=1$. Then for any $F(z) \in \mathbb{C}[[z]]$, we have

$$
F(z)=\sum_{n=0}^{\infty} \frac{c_{n} z^{n}}{\prod_{i=0}^{n} \phi_{i}(z)}
$$

where the coefficients

$$
c_{n}=\sum_{k=0}^{n}\left[z^{k}\right]\{F(z)\} \sum_{\substack{i \leq j_{i} \\ k=j_{0} \leq j_{1} \leq j_{2} \leq \cdots \leq j_{n} \leq j_{n+1}=n}} \prod_{i=0}^{n}\left[z^{j_{i+1}-j_{i}}\right]\left\{\phi_{i}(z)\right\} .
$$


For further information on Lemma 1.4, we refer the reader to 10. As for the classical Lagrange-Bürmann inversion formula the reader might consult the book [2, p. 629] by G.E. Andrews, R. Askey, and R. Roy. For its various $q$-analogues, we refer the reader to the paper 1 by G.E. Andrews, 3 by L. Carlitz, 8 by I. Gessel and D. Stanton, and [9] by Ch. Krattenthaler, especially to the good survey [13] of D. Stanton for a more comprehensive information.

A simple expression of the coefficients $c_{n}$ seems unlikely under the case (1.6a). Without doubt, such an explicit formula is the key step to successful use of this expansion formula. But in contrast, as far as (1.5) is concerned, we are able to establish the following explicit expression of $c_{n}$ via the use of matrix inversions (see Definition 2.1 below). It is just the theme of the present paper.

Theorem 1.5. For $F(z) \in \mathbb{C}[[z]]$, there exists the series expansion

$$
F(z)=\sum_{n=0}^{\infty} c_{n} z^{n} \frac{(a z ; q)_{n}}{(b z ; q)_{n}}
$$

with the coefficients

$$
c_{n}=\left[z^{n}\right]\left\{F(z) \frac{(b z ; q)_{n-1}}{(a z ; q)_{n}}\right\}-a \sum_{k=0}^{n-1} B_{n-k, 1}(a, b) q^{(n-k) k}\left[z^{k}\right]\left\{F(z) \frac{(b z ; q)_{k}}{(a z ; q)_{k+1}}\right\},
$$

where $B_{n, 1}(a, b)$ are given by

$$
z=\sum_{n=1}^{\infty} B_{n, 1}(a, b) z^{n} \frac{(a z ; q)_{n}}{(b z ; q)_{n}}
$$

As a direct application of this expansion formula, we further set up a general transformation concerning the Rogers-Fine identity (1.3).

Theorem 1.6. For $G(z)=\sum_{n=0}^{\infty} t_{n} z^{n} \in \mathbb{C}[[z]]$, it always holds

$$
\begin{aligned}
\frac{(a z ; q)_{\infty}}{(b z ; q)_{\infty}} G(z)=\sum_{n=0}^{\infty} & \frac{(a q / b, a z ; q)_{n}}{(q, b z ; q)_{n}}(b z)^{n} q^{n(n-1)} \\
& \times\left(\widetilde{G}\left(z q^{n} ; a, b\right)-a z q^{2 n} \widetilde{G}\left(z q^{n+1} ; a / q, b / q\right)\right)
\end{aligned}
$$

where

$$
\widetilde{G}(z ; a, b):=\sum_{n=0}^{\infty} t_{n} z^{n} \frac{(a z ; q)_{n}}{(b z ; q)_{n}}
$$

The rest of this paper is organized as follows. In Section 2, we shall prove Theorem 1.5. For this purpose, a series of preliminary results will be established. Section 3 is devoted to the proof of Theorem 1.6. Some applications of these two theorems to $q$-series are further discussed. Among these applications, there is a new partial theta function identity and a coefficient identity of Ramanujan's ${ }_{1} \psi_{1}$ summation formula. 


\section{The Proof of Theorem 1.5}

In this section, we proceed to show Theorem 1.5 which amounts to finding the coefficients $c_{n}$. For this purpose, we need the concept of matrix inversions and a series of preliminary lemmas.

Definition 2.1. (cf. [12, Chapters 2 and 3] or [5, Definition 3.1.1]) A pair of infinite lower-triangular matrices $A=\left(A_{n, k}\right)$ and $B=\left(B_{n, k}\right)$ is said to be inverses to each other if and only if for any integers $n, k \geq 0$,

$$
\sum_{i=k}^{n} A_{n, i} B_{i, k}=\sum_{i=k}^{n} B_{n, i} A_{i, k}= \begin{cases}0, & n \neq k \\ 1, & n=k\end{cases}
$$

where $A_{n, k}=B_{n, k}=0$ if $n<k$. As usual, we also say that $A$ and $B$ are invertible and write $A^{-1}$ for $B$.

Consider now a particular matrix $A=\left(A_{n, k}\right)$ with the entries $A_{n, k}$ given by

$$
z^{k} \frac{(a z ; q)_{k}}{(b z ; q)_{k}}=\sum_{n=k}^{\infty} A_{n, k} z^{n} .
$$

It is easy to check that $A=\left(A_{n, k}\right)$ is invertible. In what follows, let us assume its inverse

$$
A^{-1}=\left(B_{n, k}(a, b)\right)
$$

As such, we see that (2.2) is equivalent to

$$
z^{k}=\sum_{n=k}^{\infty} B_{n, k}(a, b) z^{n} \frac{(a z ; q)_{n}}{(b z ; q)_{n}} .
$$

Next, we shall focus on two kinds of generating functions of the entries $B_{n, k}(a, b)$ of the matrix $A^{-1}$.

Lemma 2.2. Let $B_{n, k}(a, b)$ be the same as above. Then we have

$$
B_{n, k+1}(a, b)+(b-a) \sum_{i=k+2}^{n} b^{i-k-2} B_{n, i}(a, b)=q^{n-k-1} B_{n-1, k}(a, b) .
$$

Proof. At first, by replacing $z$ by $z q$ in (2.3), we have

$$
(z q)^{k}=\sum_{n=k}^{\infty} B_{n, k}(a, b)(z q)^{n} \frac{(a z q ; q)_{n}}{(b z q ; q)_{n}} .
$$

Multiplying both sides with $z(1-a z) /(1-b z)$ and shifting $n$ to $n-1$, we obtain

$$
q^{k} z^{k+1}\left(1+(b-a) z \sum_{i=0}^{\infty} b^{i} z^{i}\right)=\sum_{n=k+1}^{\infty} B_{n-1, k}(a, b) q^{n-1} z^{n} \frac{(a z ; q)_{n}}{(b z ; q)_{n}} .
$$


Viewing (2.5) from the definition of (2.3), we find that (2.5) can be recast as

$$
\begin{aligned}
& q^{k} \sum_{n=k+1}^{\infty} B_{n, k+1}(a, b) z^{n} \frac{(a z ; q)_{n}}{(b z ; q)_{n}} \\
& +(b-a) q^{k} \sum_{i=0}^{\infty} b^{i} \sum_{n=k+i+2}^{\infty} B_{n, k+i+2}(a, b) z^{n} \frac{(a z ; q)_{n}}{(b z ; q)_{n}} \\
& =\sum_{n=k+1}^{\infty} B_{n-1, k}(a, b) q^{n-1} z^{n} \frac{(a z ; q)_{n}}{(b z ; q)_{n}} .
\end{aligned}
$$

Thus, by the uniqueness of the coefficients under the base $\left\{z^{n}(a z ; q)_{n} /(b z ; q)_{n}\right\}_{n=0}^{\infty}$, it holds

$$
q^{k} B_{n, k+1}(a, b)+(b-a) q^{k} \sum_{i=0}^{n-k-2} b^{i} B_{n, k+i+2}(a, b)=q^{n-1} B_{n-1, k}(a, b) .
$$

After slight simplification, we obtain

$$
B_{n, k+1}(a, b)+(b-a) \sum_{i=k+2}^{n} b^{i-k-2} B_{n, i}(a, b)=q^{n-k-1} B_{n-1, k}(a, b) .
$$

Hence (2.4) follows.

By use of Lemma 2.2. it is easy to set up a bivariate generating function of $\left\{B_{n, k}(a, b)\right\}_{n \geq k \geq 0}$.

Lemma 2.3. Let $B_{n, k}(a, b)$ be defined by (2.3). Then we have

$$
G(y, z)=\sum_{n=0}^{\infty} \frac{(b / y ; q)_{n}}{(a / y ; q)_{n}}(y z)^{n}+\sum_{n=0}^{\infty}\left(b z q^{n}-a G_{1}\left(z q^{n}\right)\right) \frac{(b / y ; q)_{n}}{(a / y ; q)_{n+1}}(y z)^{n},
$$

where

$$
\begin{aligned}
G(y, z) & :=\sum_{k=0}^{\infty} G_{k}(z) y^{k} \\
G_{k}(z) & :=\sum_{n=k}^{\infty} B_{n, k}(a, b) z^{n} .
\end{aligned}
$$

Proof. It suffices to multiply both sides of (2.4) with $b$. Then we get

$$
b B_{n, k+1}(a, b)+(b-a) \sum_{i=k+2}^{n} b^{i-k-1} B_{n, i}(a, b)=b q^{n-k-1} B_{n-1, k}(a, b) .
$$

Shifting $k$ to $k-1$ in (2.4) gives rise to

$$
B_{n, k}(a, b)+(b-a) \sum_{i=k+1}^{n} b^{i-k-1} B_{n, i}(a, b)=q^{n-k} B_{n-1, k-1}(a, b) .
$$

By abstracting (2.10) from (2.11), we come up with

$$
B_{n, k}(a, b)-a B_{n, k+1}(a, b)=q^{n-k} B_{n-1, k-1}(a, b)-b q^{n-k-1} B_{n-1, k}(a, b) .
$$


After multiplying (2.12) by $z^{n}$ and summing over $n$ for $n \geq k$, then we have

$$
\begin{aligned}
\sum_{n=k}^{\infty} B_{n, k}(a, b) z^{n} & -a \sum_{n=k}^{\infty} B_{n, k+1}(a, b) z^{n} \\
& =z q^{1-k} \sum_{n=k}^{\infty} B_{n-1, k-1}(a, b)(q z)^{n-1}-b z q^{-k} \sum_{n=k}^{\infty} B_{n-1, k}(a, b)(q z)^{n-1} .
\end{aligned}
$$

In terms of $G_{k}(z)$ defined by (2.9), this relation can be expressed as

$$
G_{k}(z)-a G_{k+1}(z)=z q^{1-k} G_{k-1}(q z)-b z q^{-k} G_{k}(q z) .
$$

Then, by multiplying $y^{k+1}$ and then summing over $k$ for $k \geq 1$ on both sides of (2.13), we further obtain

$$
(y-a) G(y, z)+(a-y) G_{0}(z)+a y G_{1}(z)=y z(y-b) G(y / q, q z)+b y z G_{0}(q z),
$$

where $G(y, z)$ is given by (2.8). Observe that $G_{0}(z)=1$. Then

$$
(y-a) G(y, z)-y z(y-b) G(y / q, q z)=y-a+b y z-a y G_{1}(z),
$$

namely,

$$
G(y, z)-y z \frac{1-b / y}{1-a / y} G(y / q, z q)=d(y, z),
$$

where, for clarity, we define

$$
d(y, z):=1+\frac{y}{y-a}\left(b z-a G_{1}(z)\right) .
$$

Setting $(y, z) \rightarrow\left(y / q^{n}, z q^{n}\right)$ in (2.14) , we obtain its equivalent version as below

$$
X(n)-y z \frac{1-b q^{n} / y}{1-a q^{n} / y} X(n+1)=d\left(y / q^{n}, z q^{n}\right),
$$

where

$$
X(n):=G\left(y / q^{n}, z q^{n}\right) .
$$

Iterating (2.15) $m$ times, we find

$$
\begin{aligned}
X(n)= & d\left(y / q^{n}, z q^{n}\right)+y z \frac{1-b q^{n} / y}{1-a q^{n} / y} X(n+1) \\
= & d\left(y / q^{n}, z q^{n}\right)+y z \frac{1-b q^{n} / y}{1-a q^{n} / y} d\left(y / q^{n+1}, z q^{n+1}\right) \\
& \quad+(y z)^{2} \frac{\left(1-b q^{n} / y\right)\left(1-b q^{n+1} / y\right)}{\left(1-a q^{n} / y\right)\left(1-a q^{n+1} / y\right)} X(n+2) \\
= & \cdots \\
= & \sum_{k=0}^{m-1} d\left(y / q^{n+k}, z q^{n+k}\right) \frac{\left(b q^{n} / y ; q\right)_{k}}{\left(a q^{n} / y ; q\right)_{k}}(y z)^{k}+\frac{\left(b q^{n} / y ; q\right)_{m}}{\left(a q^{n} / y ; q\right)_{m}}(y z)^{m} X(n+m) .
\end{aligned}
$$

Regarding the solution of this recurrence relation, we may guess and then show by induction on $m$ ( $\operatorname{set} n=0$ ) that

$$
\begin{aligned}
G(y, z) & =\sum_{k=0}^{\infty} d\left(y / q^{k}, z q^{k}\right) \frac{(b / y ; q)_{k}}{(a / y ; q)_{k}}(y z)^{k} \\
& =\sum_{k=0}^{\infty} \frac{(b / y ; q)_{k}}{(a / y ; q)_{k}}(y z)^{k}+\sum_{k=0}^{\infty} \frac{b z q^{k}-a G_{1}\left(z q^{k}\right)}{1-a q^{k} / y} \frac{(b / y ; q)_{k}}{(a / y ; q)_{k}}(y z)^{k} \\
& =\sum_{k=0}^{\infty} \frac{(b / y ; q)_{k}}{(a / y ; q)_{k}}(y z)^{k}+\sum_{k=0}^{\infty}\left(b z q^{k}-a G_{1}\left(z q^{k}\right)\right) \frac{(b / y ; q)_{k}}{(a / y ; q)_{k+1}}(y z)^{k}
\end{aligned}
$$


So the lemma is proved.

There also exists a finite univariate generating function of $\left\{B_{n, k}(a, b)\right\}_{k=0}^{n}$.

Corollary 2.4. Let $B_{n, k}(a, b)$ be defined by (2.3). Then for integer $n \geq 1$, we have

$$
\sum_{k=0}^{n} B_{n, k}(a, b) y^{k}=\frac{(b / y ; q)_{n-1}}{(a / y ; q)_{n}} y^{n}-a \sum_{k=0}^{n-1} B_{n-k, 1}(a, b) q^{(n-k) k} \frac{(b / y ; q)_{k}}{(a / y ; q)_{k+1}} y^{k} \text {. }
$$

Proof. It is an immediate consequence of Lemma 2.3. To be precise, by Lemma 2.3 , we see

$$
\begin{aligned}
G(y, z) & =\sum_{n \geq k \geq 0} B_{n, k}(a, b) y^{k} z^{n} \\
& =\sum_{n=0}^{\infty} \frac{(b / y ; q)_{n}}{(a / y ; q)_{n}}(y z)^{n}+\sum_{n=0}^{\infty}\left(b z q^{n}-a G_{1}\left(z q^{n}\right)\right) \frac{(b / y ; q)_{n}}{(a / y ; q)_{n+1}}(y z)^{n} .
\end{aligned}
$$

By equating the coefficients of $z^{n}$ on both sides, it is easy to calculate that for $n \geq 1$,

$$
\begin{aligned}
\sum_{k=0}^{n} B_{n, k}(a, b) y^{k} & =\frac{(b / y ; q)_{n}}{(a / y ; q)_{n}} y^{n}+b \frac{(b / y ; q)_{n-1}}{(a / y ; q)_{n}}(q y)^{n-1} \\
& -a \sum_{k=0}^{n} B_{n-k, 1}(a, b) q^{(n-k) k} \frac{(b / y ; q)_{k}}{(a / y ; q)_{k+1}} y^{k} \\
& =\frac{(b / y ; q)_{n-1}}{(a / y ; q)_{n}} y^{n}-a \sum_{k=0}^{n-1} B_{n-k, 1}(a, b) q^{(n-k) k} \frac{(b / y ; q)_{k}}{(a / y ; q)_{k+1}} y^{k} .
\end{aligned}
$$

The corollary is thus proved.

Remark 2.5. Evidently, the left-hand side of (2.16) is a polynomial in $y$ while the right-hand side doesn't seem that case. In fact, the coefficients $B_{n-k, 1}(a, b)$ given by (1.7c), i.e.,

$$
z=\sum_{n=1}^{\infty} B_{n, 1}(a, b) z^{n} \frac{(a z ; q)_{n}}{(b z ; q)_{n}}
$$

just satisfy

$$
S_{n}\left(a q^{k}\right)=0 \quad(0 \leq k \leq n-1),
$$

where $S_{n}(y)$ is given by

$$
\frac{S_{n}(y)}{\prod_{k=0}^{n-1}\left(y-a q^{k}\right)}:=\frac{(b / y ; q)_{n-1}}{(a / y ; q)_{n}} y^{n}-a \sum_{k=0}^{n-1} B_{n-k, 1}(a, b) q^{(n-k) k} \frac{(b / y ; q)_{k}}{(a / y ; q)_{k+1}} y^{k} .
$$

This fact guarantees that the right-hand side of (2.16) is really a polynomial in $y$.

Corollary 2.4leads us to a general matrix inversion, which will play a very crucial role in our main result, i.e., Theorem 1.5 .

Theorem 2.6 (Matrix inversion). Let $A=\left(A_{n, k}\right)$ be the infinitely lower-triangular matrix with the entries

$$
A_{n, k}=\left[z^{n-k}\right]\left\{\frac{(a z ; q)_{k}}{(b z ; q)_{k}}\right\}
$$


and assume $A^{-1}=\left(B_{n, k}(a, b)\right)$. Then

$$
B_{n, k}(a, b)=\left[z^{n-k}\right]\left\{\frac{(b z ; q)_{n-1}}{(a z ; q)_{n}}\right\}-a \sum_{i=k}^{n-1} B_{n-i, 1}(a, b) q^{(n-i) i}\left[z^{i-k}\right]\left\{\frac{(b z ; q)_{i}}{(a z ; q)_{i+1}}\right\} .
$$

Proof. It is clear that (2.19) is valid for $n=k=0$ or $k=0$. Thus we only need to show (2.19) for $n \geq 1$. To that end, we first set $y \rightarrow 1 / t$ in (2.16) and then multiply both sides with $t^{n}$. All that we obtained is

$$
\sum_{k=0}^{n} B_{n, k}(a, b) t^{n-k}=\frac{(b t ; q)_{n-1}}{(a t ; q)_{n}}-a \sum_{k=0}^{n-1} B_{n-k, 1}(a, b) \frac{(b t ; q)_{k}}{(a t ; q)_{k+1}} q^{(n-k) k} t^{n-k} .
$$

A comparison of the coefficients of $t^{n-k}$ yields

$$
\begin{aligned}
B_{n, k}(a, b) & =\left[t^{n-k}\right]\left\{\frac{(b t ; q)_{n-1}}{(a t ; q)_{n}}\right\}-a \sum_{i=0}^{n-1} B_{n-i, 1}(a, b) q^{(n-i) i}\left[t^{n-k}\right]\left\{\frac{(b t ; q)_{i}}{(a t ; q)_{i+1}} t^{n-i}\right\} \\
& =\left[t^{n-k}\right]\left\{\frac{(b t ; q)_{n-1}}{(a t ; q)_{n}}\right\}-a \sum_{i=k}^{n-1} B_{n-i, 1}(a, b) q^{(n-i) i}\left[t^{i-k}\right]\left\{\frac{(b t ; q)_{i}}{(a t ; q)_{i+1}}\right\} .
\end{aligned}
$$

Thus (2.19) is confirmed.

As byproducts of our analysis, we find two interesting properties for $\left\{B_{n, k}(a, b)\right\}_{n \geq k \geq 0}$ as follows.

Proposition 2.7. Let $B_{n, k}(a, b)$ be given by (2.3). Then for integer $n \geq 1$ and $t \in \mathbb{C}$, we have

$$
\begin{aligned}
B_{n, k}(a t, b t) & =B_{n, k}(a, b) t^{n-k} \\
{\left[z^{n}\right]\left\{\frac{(b z ; q)_{n-1}}{(a z ; q)_{n}}\right\} } & =a \sum_{i=0}^{n-1} B_{n-i, 1}(a, b) q^{(n-i) i}\left[z^{i}\right]\left\{\frac{(b z ; q)_{i}}{(a z ; q)_{i+1}}\right\} .
\end{aligned}
$$

Proof. To establish (2.21), it only needs to take $(a, b) \rightarrow(a t, b t)$ in (2.3). Then it follows

$$
z^{k}=\sum_{n=k}^{\infty} B_{n, k}(a t, b t) z^{n} \frac{(a t z ; q)_{n}}{(b t z ; q)_{n}}
$$

On the other hand, on setting $z \rightarrow t z$ in (2.3), we have

$$
z^{k}=\sum_{n=k}^{\infty} B_{n, k}(a, b) t^{n-k} z^{n} \frac{(a t z ; q)_{n}}{(b t z ; q)_{n}} .
$$

By the uniqueness of series expansion, we obtain (2.21). Identity (2.22) is a special case $k=0$ of (2.19), noting that for $n \geq 1, B_{n, 0}=0$.

After these preliminaries we are prepared to show Theorem 1.5.

Proof. The existence of (1.7a) is evident, because

$$
\left\{z^{n} \frac{(a z ; q)_{n}}{(b z ; q)_{n}} \mid n=0,1,2 \cdots\right\}
$$


is the base of $\mathbb{C}[[z]]$. Thus it only needs to evaluate the coefficients $c_{n}$ in (1.7a). To do this, by Theorem 2.6, we have

$$
\begin{aligned}
c_{n} & =\sum_{k=0}^{n}\left[z^{k}\right]\{F(z)\} B_{n, k}(a, b) \\
& =\sum_{k=0}^{n}\left[z^{k}\right]\{F(z)\}\left[z^{n-k}\right]\left\{\frac{(b z ; q)_{n-1}}{(a z ; q)_{n}}\right\} \\
& -a \sum_{i=0}^{n-1} B_{n-i, 1}(a, b) q^{(n-i) i} \sum_{k=0}^{i}\left[z^{k}\right]\{F(z)\}\left[z^{i-k}\right]\left\{\frac{(b z ; q)_{i}}{(a z ; q)_{i+1}}\right\} \\
& =\left[z^{n}\right]\left\{F(z) \frac{(b z ; q)_{n-1}}{(a z ; q)_{n}}\right\}-a \sum_{i=0}^{n-1} B_{n-i, 1}(a, b) q^{(n-i) i}\left[z^{i}\right]\left\{F(z) \frac{(b z ; q)_{i}}{(a z ; q)_{i+1}}\right\} .
\end{aligned}
$$

The conclusion is proved.

Remark 2.8. It is worth mentioning that in [6] A.M. Garsia and J. Remmel set up a $q$-Lagrange inversion formula, which asserts that for any formal power series $F(z)=\sum_{n=1}^{\infty} F_{n} z^{n}$ and $f(z)=\sum_{n=1}^{\infty} f_{n} z^{n}$ with $F_{1} f_{1} \neq 0$,

$$
\sum_{n=1}^{\infty} f_{n} F(z) F(z q) \cdots F\left(z q^{n-1}\right)=z
$$

holds if and only if

$$
\sum_{n=1}^{\infty} F_{n} f(z) f(z / q) \cdots f\left(z / q^{n-1}\right)=z .
$$

However, to the author's knowledge, it is still hard to find out explicit expressions of $f_{n}:=B_{n, 1}(a, b)$ from (2.23) even if $F(z)=z(1-a z) /(1-b z)$.

In the following, we shall examine a few specific formal expansion formulas covered by Theorem 1.5. As a first consequence, when $a=0$ we recover Carlitz's $q$-expansion formula [3, p. 206, Eq. (1.11)].

Corollary 2.9. For any $F(z) \in \mathbb{C}[[z]]$, we have

$$
F(z)=\sum_{n=0}^{\infty} \frac{c_{n} z^{n}}{(b z ; q)_{n}},
$$

where

$$
c_{n}=\left[z^{n}\right]\left\{F(z)(b z ; q)_{n-1}\right\} .
$$

We remark that Carlitz's $q$-expansion formula is a useful $q$-analogue of the Lagrange-Bürmann inversion formula. The reader may consult the survey [13] of D. Stanton concerning this topic.

A second interesting consequence occurs when $b=0$.

Corollary 2.10. Let $B_{n, 1}(a, 0)$ be given by (1.7c). Then

$$
F(z)=\sum_{n=0}^{\infty} c_{n} z^{n}(a z ; q)_{n},
$$


where the coefficients

$$
c_{n}=\left[z^{n}\right]\left\{\frac{F(z)}{(a z ; q)_{n}}\right\}-a \sum_{k=0}^{n-1} B_{n-k, 1}(a, 0) q^{(n-k) k}\left[z^{k}\right]\left\{\frac{F(z)}{(a z ; q)_{k+1}}\right\} .
$$

As a third consequence, the special case $b=a q$ leads us to

Corollary 2.11. Let $F(z)=\sum_{n=0}^{\infty} a_{n} z^{n}$ and $F_{k}(z)=\sum_{i=0}^{k} a_{i} z^{i}$, being the $k$ truncated series of $F(z)$. Suppose that

$$
\frac{F(z)}{1-a z}=\sum_{n=0}^{\infty} \frac{c_{n} z^{n}}{1-a z q^{n}}
$$

Then $c_{0}=a_{0}$ and $n \geq 1$,

$$
c_{n}=\sum_{k=0}^{n-1} g_{n-k}(q) q^{(n-k) k}\left[z^{n}\right]\left\{\frac{F(z)-F_{k}(z)}{1-a z}\right\},
$$

where $g_{n}(q)$ are polynomials in $q$ given recursively by

$$
g_{n}(q)=1-\sum_{i=1}^{n-1} g_{n-i}(q) q^{(n-i) i} .
$$

Proof. In such case, we first solve the recurrence relation (2.19) with $k=1$ for $B_{n, 1}(a, a q)$, viz.

$$
B_{n, 1}(a, a q)=a^{n-1}-\sum_{i=1}^{n-1} B_{n-i, 1}(a, a q) q^{(n-i) i} a^{i} .
$$

The solution is recursively given by

$$
\left\{\begin{array}{l}
B_{n, 1}(a, a q)=g_{n}(q) a^{n-1}, \\
g_{n}(q)=1-\sum_{i=1}^{n-1} g_{n-i}(q) q^{(n-i) i} .
\end{array}\right.
$$

By virtue of (2.28), we are now able to calculate $c_{n}$. To do this, by Theorem 1.5 we have

$$
\begin{aligned}
c_{n} & =\left[z^{n}\right]\left\{\frac{F(z)}{1-a z}\right\}-\sum_{k=0}^{n-1} g_{n-k}(q) q^{(n-k) k} a^{n-k}\left[z^{k}\right]\left\{\frac{F(z)}{1-a z}\right\} \\
& =\left[z^{n}\right]\left\{\frac{F(z)}{1-a z}\right\}-\sum_{k=0}^{n-1} g_{n-k}(q) q^{(n-k) k}\left[z^{n}\right]\left\{\frac{F_{k}(z)}{1-a z}\right\} \\
& =\left[z^{n}\right]\left\{\sum_{k=0}^{n-1} g_{n-k}(q) q^{(n-k) k} \frac{F(z)-F_{k}(z)}{1-a z}\right\} .
\end{aligned}
$$

In the penultimate equality we have used the fact that

$$
a^{n-k}\left[z^{k}\right]\left\{\frac{F(z)}{1-a z}\right\}=\left[z^{n}\right]\left\{\frac{F_{k}(z)}{1-a z}\right\}
$$

and in the last equality, we have invoked (2.28) again. The conclusion is proved. 
It is also of interest to note that if $F(z)$ is a polynomial of degree $m+1$, say

$$
F(z)=(1-a z) \prod_{n=1}^{m}\left(1-t_{n} z\right)
$$

and $F_{k}(z)=F(z)$ for $k \geq m+1$, then Corollary 2.11 reduces to

Corollary 2.12. With the same notation as Corollary 2.11 Then we have

$$
\prod_{n=1}^{m}\left(1-t_{n} z\right)=\sum_{n=0}^{\infty} \frac{c_{n} z^{n}}{1-a z q^{n}}
$$

where $c_{0}=1$ and $n \geq 1$,

$$
c_{n}=\sum_{k=0}^{\min \{m, n-1\}} g_{n-k}(q) q^{(n-k) k}\left[z^{n}\right]\left\{\prod_{i=1}^{m}\left(1-t_{i} z\right)-\frac{F_{k}(z)}{1-a z}\right\} .
$$

\section{Applications to $q$-Series theory}

Unlike the preceding section, we now focus our attention on applications of Theorem 1.5 to the $q$-series theory. In this sense, we assume that all results are subject to appropriate convergent conditions of rigorous analytic theory, unless otherwise stated.

Let us begin with the proof of Theorem 1.6 ,

Proof. We only need to make use of Theorem 1.5 as well as the $q$-binomial theorem [7. (II.3)] to get

$$
\frac{(a z ; q)_{\infty}}{(b z ; q)_{\infty}} G(z)=\sum_{n=0}^{\infty} c_{n} z^{n} \frac{(a z ; q)_{n}}{(b z ; q)_{n}}=S_{1}-a S_{2},
$$

where

$$
\begin{aligned}
S_{1} & :=\sum_{n=0}^{\infty} \sum_{i=0}^{n} \frac{(a q / b ; q)_{i}}{(q ; q)_{i}} b^{i} q^{(n-1) i} t_{n-i} z^{n} \frac{(a z ; q)_{n}}{(b z ; q)_{n}} \\
S_{2} & :=\sum_{n=0}^{\infty} \sum_{k=0}^{n-1} B_{n-k, 1}(a, b) q^{(n-k) k} \sum_{i=0}^{k} \frac{(a q / b ; q)_{i}}{(q ; q)_{i}}\left(b q^{k}\right)^{i} t_{k-i} z^{n} \frac{(a z ; q)_{n}}{(b z ; q)_{n}} .
\end{aligned}
$$

After a mere series rearrangement, we get

$$
\begin{aligned}
S_{1} & =\sum_{i=0}^{\infty} \frac{(a q / b ; q)_{i}}{(q ; q)_{i}} b^{i} q^{i^{2}-i} z^{i} \frac{(a z ; q)_{i}}{(b z ; q)_{i}} \sum_{n=0}^{\infty} q^{n i} t_{n} z^{n} \frac{\left(a z q^{i} ; q\right)_{n}}{\left(b z q^{i} ; q\right)_{n}} \\
& =\sum_{i=0}^{\infty} \frac{(a q / b, a z ; q)_{i}}{(q, b z ; q)_{i}} b^{i} q^{i^{2}-i} z^{i} \widetilde{G}\left(z q^{i} ; a, b\right) .
\end{aligned}
$$

Hereafter, as given by (1.8b),

$$
\widetilde{G}(z ; a, b)=\sum_{n=0}^{\infty} t_{n} z^{n} \frac{(a z ; q)_{n}}{(b z ; q)_{n}} .
$$


In a similar way, it is easily found that

$$
S_{2}=\sum_{i=0}^{\infty} \frac{(a q / b ; q)_{i}}{(q ; q)_{i}} b^{i} \sum_{k=i}^{\infty} t_{k-i} z^{k} \frac{(a z ; q)_{k}}{(b z ; q)_{k}} q^{k i} \Delta_{k},
$$

where

$\Delta_{k}:=\sum_{n=k+1}^{\infty} B_{n-k, 1}(a, b)\left(z q^{k}\right)^{n-k} \frac{\left(a z q^{k} ; q\right)_{n-k}}{\left(b z q^{k} ; q\right)_{n-k}}=\sum_{n=1}^{\infty} B_{n, 1}(a, b)\left(z q^{k}\right)^{n} \frac{\left(a z q^{k} ; q\right)_{n}}{\left(b z q^{k} ; q\right)_{n}}=z q^{k}$.

The last equality is based on (1.7c). Therefore,

$$
\begin{aligned}
S_{2} & =z \sum_{i=0}^{\infty} \frac{(a q / b, a z ; q)_{i}}{(q, b z ; q)_{i}}\left(b z q^{i+1}\right)^{i} \sum_{k=0}^{\infty} t_{k} \frac{\left(a z q^{i} ; q\right)_{k}}{\left(b z q^{i} ; q\right)_{k}}\left(z q^{i+1}\right)^{k} \\
& =z \sum_{i=0}^{\infty} \frac{(a q / b, a z ; q)_{i}}{(q, b z ; q)_{i}}\left(b z q^{i+1}\right)^{i} \widetilde{G}\left(z q^{i+1} ; a / q, b / q\right) .
\end{aligned}
$$

Finally, we achieve

$\frac{(a z ; q)_{\infty}}{(b z ; q)_{\infty}} G(z)=\sum_{n=0}^{\infty} \frac{(a q / b, a z ; q)_{n}}{(q, b z ; q)_{n}}(b z)^{n} q^{n(n-1)}\left(\widetilde{G}\left(z q^{n} ; a, b\right)-a z q^{2 n} \widetilde{G}\left(z q^{n+1} ; a / q, b / q\right)\right)$.

This gives the complete proof of the theorem.

With regard to applications of Theorem 1.6 to $q$-series, it is necessary to set up

Corollary 3.1. For integer $r \geq 0$ and $|c z|<1$, it holds

$$
\begin{aligned}
& \frac{(a z q ; q)_{\infty}}{(b z ; q)_{\infty}}{ }_{r+1} \phi_{r}\left[\begin{array}{c}
A_{1}, A_{2}, \ldots, A_{r+1} \\
B_{1}, B_{2}, \ldots, B_{r}
\end{array} ;, c z\right] \\
& =\lim _{x, y \rightarrow \infty} \sum_{n=0}^{\infty} \frac{\left(a z,(a z)^{1 / 2} q,-(a z)^{1 / 2} q, a q / b, x, y ; q\right)_{n}}{\left(q,(a z)^{1 / 2},-(a z)^{1 / 2}, b z, a z q / x, a z q / y ; q\right)_{n}}\left(\frac{b z}{x y}\right)^{n} \\
& \quad \times{ }_{r+3} \phi_{r+2}\left[\begin{array}{c}
a z q^{n}, a z q^{2 n+1}, A_{1}, A_{2}, \ldots, A_{r+1} \\
b z q^{n}, a z q^{2 n}, B_{1}, B_{2}, \ldots, B_{r}
\end{array} ;, c z q^{n}\right] .
\end{aligned}
$$

Proof. It suffices to set in Theorem 1.6

$$
G(z)={ }_{r+1} \phi_{r}\left[\begin{array}{c}
A_{1}, A_{2}, \ldots, A_{r+1} \\
B_{1}, B_{2}, \ldots, B_{r}
\end{array} ; q, c z\right]
$$

which means

$$
t_{k}=\frac{\left(A_{1}, A_{2}, \ldots, A_{r+1} ; q\right)_{k}}{\left(q, B_{1}, B_{2}, \ldots, B_{r} ; q\right)_{k}} c^{k}
$$

In the sequel, it is routine to compute

$$
\begin{aligned}
H_{n}(z ; a, b) & :=\frac{\widetilde{G}\left(z q^{n} ; a, b\right)-a z q^{2 n} \widetilde{G}\left(z q^{n+1} ; a / q, b / q\right)}{1-a z q^{2 n}} \\
& =\sum_{k=0}^{\infty} \frac{\left(a z q^{n}, a z q^{2 n+1} ; q\right)_{k}}{\left(b z q^{n}, a z q^{2 n} ; q\right)_{k}}\left(z q^{n}\right)^{k} t_{k} \\
& ={ }_{r+3} \phi_{r+2}\left[\begin{array}{c}
a z q^{n}, a z q^{2 n+1}, A_{1}, A_{2}, \ldots, A_{r+1} \\
b z q^{n}, a z q^{2 n}, B_{1}, B_{2}, \ldots, B_{r}
\end{array} ; q, c z q^{n}\right] .
\end{aligned}
$$


This reduces (1.8a) of Theorem 1.6 to

$$
\begin{aligned}
& {\frac{(a z q ; q)_{\infty}}{(b z ; q)_{\infty}}}_{r+1} \phi_{r}\left[\begin{array}{c}
A_{1}, A_{2}, \ldots, A_{r+1} \\
B_{1}, B_{2}, \ldots, B_{r}
\end{array} ;, c z\right] \\
& \quad=\sum_{n=0}^{\infty} \frac{(a q / b, a z ; q)_{n}}{(q, b z ; q)_{n}}(b z)^{n} q^{n(n-1)} \frac{1-a z q^{2 n}}{1-a z} H_{n}(z ; a, b) .
\end{aligned}
$$

Finally, using the basic relations

$$
\frac{1-a z q^{2 n}}{1-a z}=\frac{\left((a z)^{1 / 2} q,-(a z)^{1 / 2} q ; q\right)_{n}}{\left((a z)^{1 / 2},-(a z)^{1 / 2} ; q\right)_{n}}
$$

and

$$
\lim _{x, y \rightarrow \infty} \frac{(x, y ; q)_{n}}{(a z q / x, a z q / y ; q)_{n}}\left(\frac{1}{x y}\right)^{n}=q^{n(n-1)},
$$

we derive (3.1) from (3.2) directly.

The following are two special instances of Theorem 1.6

Example 3.2. The following transformation formulas are valid.

$$
\begin{aligned}
& \frac{(a z ; q)_{\infty}}{(b z ; q)_{\infty}}= \sum_{n=0}^{\infty} \frac{(a q / b, a z ; q)_{n}}{(q, b z ; q)_{n}}(b z)^{n} q^{n(n-1)}\left(1-a z q^{2 n}\right) \\
& \frac{(a z q, A B z ; q)_{\infty}}{(b z, B z ; q)_{\infty}}=\sum_{n=0}^{\infty} \frac{\left((a z)^{1 / 2} q,-(a z)^{1 / 2} q, a q / b, a z ; q\right)_{n}}{\left(q,(a z)^{1 / 2},-(a z)^{1 / 2}, b z ; q\right)_{n}}(b z)^{n} q^{n(n-1)} \\
& \quad \times{ }_{3} \phi_{2}\left[\begin{array}{c}
a z q^{n}, a z q^{2 n+1}, A \\
b z q^{n}, a z q^{2 n}
\end{array} ;, B z q^{n}\right]
\end{aligned}
$$

Proof. Identity (3.3) comes from $G(z)=1$ in Theorem 1.6 and (3.4) does by taking $G(z)=(A B z ; q)_{\infty} /(B z ; q)_{\infty}$, i.e., $t_{k}=(A ; q)_{k} /(q ; q)_{k} B^{k}$ in Theorem 1.6 or $r=0$ in Corollary 3.1

The next conclusion shows how Theorem [1.6 can be applied to known transformation formulas for finding new results.

Corollary 3.3. For $|z|<1$, we have

$$
\begin{aligned}
{ }_{2} \phi_{1}\left[\begin{array}{c}
A, B \\
C
\end{array} ;, z\right]= & \sum_{n=0}^{\infty} \frac{(A B q / C, A B z / C ; q)_{n}}{(q, z ; q)_{n}} z^{n} q^{n(n-1)}\left(1-\frac{A B z q^{2 n}}{C}\right) \\
& \times{ }_{4} \phi_{3}\left[\begin{array}{c}
A B z q^{n} / C, A B z q^{2 n+1} / C, C / A, C / B \\
C, z q^{n}, A B z q^{2 n} / C
\end{array} ;, \frac{A B z q^{n}}{C}\right] .
\end{aligned}
$$

Proof. Performing as above, we choose in Theorem 1.6

$$
G(z)={ }_{2} \phi_{1}\left[\begin{array}{c}
C / A, C / B \\
C
\end{array} ;, \frac{A B z}{C}\right],
$$

which corresponds to

$$
t_{k}=\frac{(C / A, C / B ; q)_{k}}{(q, C ; q)_{k}}\left(\frac{A B}{C}\right)^{k} .
$$


In this case, it is clear that

$$
\begin{aligned}
H_{n}(z ; a, b): & =\frac{\widetilde{G}\left(z q^{n} ; a, b\right)-a z q^{2 n} \widetilde{G}\left(z q^{n+1} ; a / q, b / q\right)}{1-a z q^{2 n}} \\
& ={ }_{4} \phi_{3}\left[\begin{array}{c}
a z q^{n}, a z q^{2 n+1}, C / A, C / B \\
C, b z q^{n}, a z q^{2 n}
\end{array} ;, \frac{A B z q^{n}}{C}\right] .
\end{aligned}
$$

As a result, from Theorem 1.6 it follows

$\frac{(a z ; q)_{\infty}}{(b z ; q)_{\infty}}{ }_{2} \phi_{1}\left[\begin{array}{c}C / A, C / B \\ C\end{array} ;, \frac{A B z}{C}\right]=\sum_{n=0}^{\infty} \frac{(a q / b, a z ; q)_{n}}{(q, b z ; q)_{n}}(b z)^{n} q^{n(n-1)}\left(1-a z q^{2 n}\right) H_{n}(z ; a, b)$.

In this form, taking $a=A B / C$ and $b=1$, we obtain

$$
\begin{aligned}
& \frac{(A B z / C ; q)_{\infty}}{(z ; q)_{\infty}}{ }_{2} \phi_{1}\left[\begin{array}{c}
C / A, C / B \\
C
\end{array} ;, \frac{A B z}{C}\right] \\
& =\sum_{n=0}^{\infty} \frac{(A B q / C, A B z / C ; q)_{n}}{(q, z ; q)_{n}} z^{n} q^{n(n-1)}\left(1-A B z q^{2 n} / C\right) H_{n}(z ; A B / C, 1) .
\end{aligned}
$$

By combining (3.6) with Heine's third transformation [7, (III.3)]

$$
{ }_{2} \phi_{1}\left[\begin{array}{c}
A, B \\
C
\end{array} ; q, z\right]=\frac{(A B z / C ; q)_{\infty}}{(z ; q)_{\infty}}{ }_{2} \phi_{1}\left[\begin{array}{c}
C / A, C / B \\
C
\end{array} ; q, \frac{A B z}{C}\right],
$$

then we reformulate (3.6) in standard notation of $q$-series as

$$
\begin{aligned}
{ }_{2} \phi_{1}\left[\begin{array}{c}
A, B \\
C
\end{array} ;, z\right]= & \sum_{n=0}^{\infty} \frac{(A B q / C, A B z / C ; q)_{n}}{(q, z ; q)_{n}} z^{n} q^{n(n-1)}\left(1-\frac{A B z q^{2 n}}{C}\right) \\
& \times{ }_{4} \phi_{3}\left[\begin{array}{c}
A B z q^{n} / C, A B z q^{2 n+1} / C, C / A, C / B \\
C, z q^{n}, A B z q^{2 n} / C
\end{array} ;, \frac{A B z q^{n}}{C}\right] .
\end{aligned}
$$

The conclusion is proved.

Perhaps, the most interesting case is the following partial theta function identity. It can be derived from Theorem 1.6 with the help of two Coogan-Ono type identities (1.2) and (1.4).

Corollary 3.4 (Partial theta function identity). Let $\theta(z ; q)$ be the partial theta function given by

$$
\sum_{n=0}^{\infty}(-1)^{n} q^{n(n-1) / 2} z^{n}
$$

Then

$$
\begin{aligned}
& \frac{(z q ; q)_{\infty}}{(-z q ; q)_{\infty}}+\sum_{n=0}^{\infty} \frac{(-1, z ; q)_{n}}{(q,-z q ; q)_{n}}(-z)^{n} q^{n^{2}+n} \\
& \quad=\sum_{n=0}^{\infty} \frac{(-1, z ; q)_{n}}{(q,-z q ; q)_{n}}\left(1+q^{n}+z q^{n}-z q^{2 n}\right)(-z)^{n} q^{n^{2}} \theta\left(z^{2} q^{2 n+1} ; q^{2}\right)
\end{aligned}
$$

Proof. Recall that Lemma 1.1 gives

$$
\sum_{k=0}^{\infty} z^{k} \frac{(z ; q)_{k}}{(-z q ; q)_{k}}=(1+z) \sum_{k=0}^{\infty}(-1)^{k} z^{2 k} q^{k^{2}} .
$$


Lemma 1.3 can be restated as

$$
\sum_{k=0}^{\infty} z^{k} \frac{(z ; q)_{k}}{(-z q ; q)_{k}}\left(1-z q^{k}\right)=1+2 \sum_{k=1}^{\infty}(-1)^{k} z^{2 k} q^{k^{2}} .
$$

Subtracting (3.9) from (3.8), we obtain

$$
\begin{gathered}
z \sum_{k=0}^{\infty}(q z)^{k} \frac{(z ; q)_{k}}{(-z q ; q)_{k}}=z+(z-1) \sum_{k=1}^{\infty}(-1)^{k} z^{2 k} q^{k^{2}}, \\
\text { i.e., } \sum_{k=0}^{\infty}(q z)^{k} \frac{(z ; q)_{k}}{(-z q ; q)_{k}}=\sum_{k=0}^{\infty}(-1)^{k} z^{2 k} q^{k^{2}}-\sum_{k=1}^{\infty}(-1)^{k} z^{2 k-1} q^{k^{2}} .
\end{gathered}
$$

Using (3.8) and (3.10), as well as referring to (1.8b) with $t_{k}=1$, we thus obtain

$$
\begin{aligned}
\widetilde{G}(z ; 1,-q) & =(1+z) \sum_{k=0}^{\infty}(-1)^{k} z^{2 k} q^{k^{2}}, \\
\widetilde{G}(z q ; 1 / q,-1) & =\sum_{k=0}^{\infty}(-1)^{k} z^{2 k} q^{k^{2}}-\sum_{k=1}^{\infty}(-1)^{k} z^{2 k-1} q^{k^{2}} .
\end{aligned}
$$

Thus it is easy to check that

$$
\begin{aligned}
& \widetilde{G}\left(z q^{n} ; 1,-q\right)-z q^{2 n} \widetilde{G}\left(z q^{n+1} ; 1 / q,-1\right) \\
& =\left(1+z q^{n}\right) \sum_{k=0}^{\infty}(-1)^{k} z^{2 k} q^{k^{2}+2 k n}-z q^{2 n} \sum_{k=0}^{\infty}(-1)^{k} z^{2 k} q^{k^{2}+2 k n}+q^{n} \sum_{k=1}^{\infty}(-1)^{k} z^{2 k} q^{k^{2}+2 k n} \\
& =-q^{n}+\left(1+q^{n}+z q^{n}-z q^{2 n}\right) \sum_{k=0}^{\infty}(-1)^{k} z^{2 k} q^{k^{2}+2 k n} .
\end{aligned}
$$

Note that the summation on the right-hand side can be recast in terms of $\theta(z ; q)$. We thus obtain

$\widetilde{G}\left(z q^{n} ; 1,-q\right)-z q^{2 n} \widetilde{G}\left(z q^{n+1} ; 1 / q,-1\right)=-q^{n}+\left(1+q^{n}+z q^{n}-z q^{2 n}\right) \theta\left(z^{2} q^{2 n+1} ; q^{2}\right)$.

This reduces the whole equation (1.8a) to

$$
\begin{aligned}
\frac{(z q ; q)_{\infty}}{(-z q ; q)_{\infty}} & +\sum_{n=0}^{\infty} \frac{(-1, z ; q)_{n}}{(q,-z q ; q)_{n}}(-z)^{n} q^{n^{2}+n} \\
& =\sum_{n=0}^{\infty} \frac{(-1, z ; q)_{n}}{(q,-z q ; q)_{n}}(-z)^{n} q^{n^{2}}\left(1+q^{n}+z q^{n}-z q^{2 n}\right) \theta\left(z^{2} q^{2 n+1} ; q^{2}\right) .
\end{aligned}
$$

Thus Identity (3.7) is proved.

In the case that $z=q^{-m}, m \geq 1$, (3.7) reduces to a finite summation of $\theta(z ; q)$.

Example 3.5. For $m \geq 1$, we have

$$
\begin{aligned}
& \sum_{n=0}^{m} \frac{(-1 ; q)_{n}}{\left(-q^{1-m} ; q\right)_{n}}\left[\begin{array}{c}
m \\
n
\end{array}\right]_{q} q^{3 n^{2} / 2+n / 2-2 n m} \\
& =\sum_{n=0}^{m} \frac{(-1 ; q)_{n}}{\left(-q^{1-m} ; q\right)_{n}}\left[\begin{array}{c}
m \\
n
\end{array}\right]_{q} q^{3 n^{2} / 2-n / 2-2 n m}\left(1+q^{n}+q^{n-m}-q^{2 n-m}\right) \theta\left(q^{2 n-2 m+1} ; q^{2}\right),
\end{aligned}
$$

where $\left[\begin{array}{c}m \\ n\end{array}\right]_{q}$ is the usual $q$-binomial coefficient. 
Proof. It suffices to take $z=q^{-m}$ in (3.7) and simplify the obtained by using the facts that for integer $m \geq 1,\left(q^{1-m} ; q\right)_{\infty}=0$ and

$$
\frac{\left(q^{-m} ; q\right)_{n}}{(q ; q)_{n}}=\left[\begin{array}{c}
m \\
n
\end{array}\right]_{q}(-1)^{n} q^{n(n-1) / 2-m n}
$$

It would be natural to expect that Theorem 1.5 can be applied to bilateral $q$-series. The reader is referred to [7, Eq. (5.1.2)] or (1.1) for the definition of bilateral $q$-series. As an interesting example, we now set up a coefficient identity of the famous Ramanujan ${ }_{1} \psi_{1}$ summation formula [7, (II.29)].

Corollary 3.6. Let $B_{n, 1}(a, b)$ be given by (1.7c). For $|b / a|<|z|<1$, and integer $n \geq 0$, it holds

$$
\begin{aligned}
& {\left[z^{n}\right]\left\{\frac{\left(a q z^{2}, 1 / a z^{2} ; q\right)_{\infty}}{\left(z, b / a z, b q^{n} z, 1 / a z ; q\right)_{\infty}(a q z ; q)_{n}}\right\}=\frac{1}{(q, b / a ; q)_{\infty}}} \\
& \quad+a q \sum_{k=0}^{n-1} B_{n-k, 1}(a q, b q) q^{(n-k) k}\left[z^{k}\right]\left\{\frac{\left(a q z^{2}, 1 / a z^{2} ; q\right)_{\infty}}{\left(z, b / a z, b q^{k+1} z, 1 / a z ; q\right)_{\infty}(a q z ; q)_{k+1}}\right\} .
\end{aligned}
$$

Proof. Observe that Ramanujan's ${ }_{1} \psi_{1}$ sum states that for $|b / a|<|z|<1$,

$$
\sum_{k=-\infty}^{\infty} \frac{(a ; q)_{k}}{(b ; q)_{k}} z^{k}=\frac{(a z, q / a z, q, b / a ; q)_{\infty}}{(z, b / a z, b, q / a ; q)_{\infty}} .
$$

Set $(a, b) \rightarrow(a q z, b q z)$. Then we arrive at

$$
\sum_{k=-\infty}^{\infty} \frac{(a q z ; q)_{k}}{(b q z ; q)_{k}} z^{k}=\frac{\left(a q z^{2}, 1 / a z^{2}, q, b / a ; q\right)_{\infty}}{(z, b / a z, b q z, 1 / a z ; q)_{\infty}}
$$

which can be reformulated in the form

$$
f(z)+g(1 / z)=F(z),
$$

where we define

$$
\begin{aligned}
f(z) & :=\sum_{k=0}^{\infty} \frac{(a q z ; q)_{k}}{(b q z ; q)_{k}} z^{k}, g(z):=\sum_{k=1}^{\infty} \frac{(z / b ; q)_{k}}{(z / a ; q)_{k}}\left(\frac{b z}{a}\right)^{k}, \\
F(z) & :=\frac{\left(a q z^{2}, 1 / a z^{2}, q, b / a ; q\right)_{\infty}}{(z, b / a z, b q z, 1 / a z ; q)_{\infty}} .
\end{aligned}
$$

Now we apply the expansion formula in Theorem 1.5 to $f(z)$. It follows from (1.7b) that for $n \geq 0$,

$$
1=\left[z^{n}\right]\left\{f(z) \frac{(b q z ; q)_{n-1}}{(a q z ; q)_{n}}\right\}-a q \sum_{k=0}^{n-1} B_{n-k, 1}(a q, b q) q^{(n-k) k}\left[z^{k}\right]\left\{f(z) \frac{(b q z ; q)_{k}}{(a q z ; q)_{k+1}}\right\} .
$$

Next, observe that

$$
\left[z^{n}\right]\left\{f(z) \frac{(b q z ; q)_{n-1}}{(a q z ; q)_{n}}\right\}=\sum_{k=0}^{n}\left[z^{k}\right]\{f(z)\} \times\left[z^{n-k}\right]\left\{\frac{(b q z ; q)_{n-1}}{(a q z ; q)_{n}}\right\},
$$

while for $k \geq 0$, due to (3.17), it holds

$$
\left[z^{k}\right]\{f(z)\}=\left[z^{k}\right]\{F(z)\} .
$$


We immediately obtain

$$
\left[z^{k}\right]\left\{f(z) \frac{(b q z ; q)_{k-1}}{(a q z ; q)_{k}}\right\}=\left[z^{k}\right]\left\{F(z) \frac{(b q z ; q)_{k-1}}{(a q z ; q)_{k}}\right\} .
$$

This simplifies (3.18) to

$$
\begin{aligned}
1 & =\left[z^{n}\right]\left\{F(z) \frac{(b q z ; q)_{n-1}}{(a q z ; q)_{n}}\right\}-a q \sum_{k=0}^{n-1} B_{n-k, 1}(a q, b q) q^{(n-k) k}\left[z^{k}\right]\left\{F(z) \frac{(b q z ; q)_{k}}{(a q z ; q)_{k+1}}\right\} \\
& =\left[z^{n}\right]\left\{\frac{\left(a q z^{2}, 1 / a z^{2}, q, b / a ; q\right)_{\infty}}{\left(z, b / a z, b q^{n} z, 1 / a z ; q\right)_{\infty}(a q z ; q)_{n}}\right\} \\
& -a q \sum_{k=0}^{n-1} B_{n-k, 1}(a q, b q) q^{(n-k) k}\left[z^{k}\right]\left\{\frac{\left(a q z^{2}, 1 / a z^{2}, q, b / a ; q\right)_{\infty}}{\left(z, b / a z, b q^{k+1} z, 1 / a z ; q\right)_{\infty}(a q z ; q)_{k+1}}\right\} .
\end{aligned}
$$

It turns out to be (3.14).

We conclude our paper with a coefficient identity of the Coogan-Ono identity (1.2) which can be easily derived by using (2.3).

Corollary 3.7. Let $B_{n, k}(a, b)$ be given by (2.3). Then for any integer $n \geq 0$, we have

$$
\sum_{k=0}^{\lfloor n / 2\rfloor} B_{n, 2 k}(1,-q)(-1)^{k} q^{k^{2}}+\sum_{k=0}^{\lfloor(n-1) / 2\rfloor} B_{n, 2 k+1}(1,-q)(-1)^{k} q^{k^{2}}=1,
$$

where $\lfloor x\rfloor$ denotes the usual floor function.

Proof. It suffices to take $a=1, b=-q$ in Theorem 1.5 and

$$
F(z)=(1+z) \sum_{n=0}^{\infty}(-1)^{n} z^{2 n} q^{n^{2}}:=\sum_{n=0}^{\infty} a_{n} z^{n}
$$

So we are back with the series expansion

$$
F(z)=\sum_{n=0}^{\infty} z^{n} \frac{(z ; q)_{n}}{(-z q ; q)_{n}} .
$$

Thus, by (2.3) instead of (1.7b), we obtain

$$
\begin{aligned}
1=\sum_{k=0}^{n} B_{n, k}(1,-q) a_{k} & =\sum_{2 k=0}^{n} B_{n, 2 k}(1,-q)(-1)^{k} q^{k^{2}} \\
& +\sum_{2 k+1=1}^{n} B_{n, 2 k+1}(1,-q)(-1)^{k} q^{k^{2}} .
\end{aligned}
$$

\section{ACKNOWLEDGEMENTS}

This work was supported by the National Natural Science Foundation of China [Grant No. 11471237]. 


\section{REFERENCES}

1. Andrews, G.E.: Identities in Combinatorics. II: A $q$-analogue of the Lagrange inversion theorem, Proc. Amer. Math. Soc. 53, 240-245(1975)

2. Andrews, G.E., Askey, R., Roy, R.: Special Functions, Encyclopedia of Mathematics and Its Applications, Vol. 71, Cambridge University Press, Cambridge, UK(1999)

3. Carlitz, L.: Some $q$-expansion theorems, Glas. Math. Ser. III 8(28), 205-214(1973)

4. Coogan, G.H., Ono, K.: A $q$-series identity and the arithmetic of Hurwitz zeta-functions, Proc. Amer. Math. Soc. 131, 719-724(2003)

5. Egorychev, G.P.: Integral Representation and the Computation of Combinatorial Sums, Amer. Math. Soc. Translations, Vol. 59, (1984)

6. Garsia, A.M., Remmel,J.: A novel form of $q$-Lagrange inversion, Houston J. Math. 12, 503523(1986)

7. Gasper, G., Rahman, M.: Basic Hypergeometric Series, 2nd edition, Cambridge University Press (2004)

8. Gessel, I., Stanton, D.: Applications of $q$-Lagrange inversion to basic hypergeometric series, Trans. Amer. Math. Soc. 277, 173-203(1983)

9. Krattenthaler, Ch.: A new $q$-Lagrange formula and some applications, Proc. Amer. Math. Soc. 90, 338-344(1984)

10. Ma, X.R.: A novel extension of the Lagrange-Bürmann expansion formula, Linear Algebra Appl. 433, 2152-2160(2010)

11. Olver, F.W.J., Lozier, D.W., Boisvert, R.F., Clark, C.W.: (editors). NIST Handbook of Mathematical Functions. Cambridge University Press, New York, NY(2010). Print companion to $[\mathrm{DLMF}]$

12. Riordan, J.: Combinatorial Identities, J. Wiley\&Sons, Inc., New York-London-Sydney(1968)

13. Stanton, D.: Recent results for the $q$-Lagrange inversion formula, Ramanujan Revisited (G.E. Andrews et al., eds.), Academic Press, New York, pp. 525-536(1988) 